\title{
Perfil de mortalidade dos pacientes internados em uma Unidade de Terapia Intensiva (UTI) adulto em um hospital universitário do Distrito Federal
}

\author{
Mortality profile of patients admitted na adult Intensive Care Unit (ICU) in a University \\ Hospital in the Federal District
}

Perfil de mortalidad de pacientes ingresados en una Unidad de Cuidados Intensivos (UCI) en un hospital universitario en el Distrito Federal

Ana Aurora Reis Campos Neves Ferrão ${ }^{1 *}$, Juliana Mendes Pereira ${ }^{1}$, Laura Beatriz Sousa de Jesus Martelletti ${ }^{1}$, Luísa Resende Martinello ${ }^{1}$, Larissa Cristine Guimarães dos Santos ${ }^{1}$, Carla Targino Bruno dos Santos ${ }^{1}$, Keila Cristianne Trindade da Cruz ${ }^{1}$.

\section{RESUMO}

Objetivo: Identificar o perfil sociodemográfico e de saúde dos pacientes que evoluíram a óbito na Unidade de Terapia Intensiva (UTI) de um hospital universitário. Métodos: Estudo transversal, retrospectivo, descritivo, com abordagem quantitativa, baseado em dados secundários extraídos em análise de prontuários. A amostra foi composta por todos os prontuários de pacientes que evoluíram a óbito na UTI durante 0 ano de 2015 $(n=40)$. Resultados: Constatou-se que $20,7 \%$ dos pacientes internados evoluíram para óbito, em sua maioria idosos $(67,5 \%)$, do sexo masculino (67,5\%); 30\% eram provenientes do centro cirúrgico, com média de 22,6 dias de internação; $75 \%$ dos diagnósticos de óbito foram sepse/choque séptico, apresentaram em média 6,3 procedimentos invasivos por paciente, $80 \%$ tinham relato de lesão em pele e $75 \%$ fizeram uso de drogas vasoativas, principalmente a Noradrenalina $(72,5 \%)$. Conclusão: O conhecimento do perfil sociodemográfico e de saúde dessas pessoas é fundamental para os profissionais que trabalham em uma UTI, pois, por meio das informações específicas, podem traçar estratégias que possibilitem um cuidado adequado e de qualidade para a população, em sua especificidade.

Palavras-chave: Unidades de Terapia Intensiva, Mortalidade, Sepse.

\section{ABSTRACT}

Objective: To identify the sociodemographic and health profile of patients who died at the Intensive Care Unit (ICU) of a university hospital. Method: Transversal, retrospective, descriptive study, with a quantitative approach, based on secondary data extracted from medical records analysis. The sample consisted of all medical records of patients who died in the ICU during the year $2015(n=40)$. Results: It was found that $20.7 \%$ of hospitalized patients died, most of them elderly $(67.5 \%)$, male $(67.5 \%) ; 30 \%$ came from the surgical center, with an average of 22.6 days of hospitalization; $75 \%$ of the diagnoses of death were sepsis / septic shock, presented on average 6.3 invasive procedures per patient, $80 \%$ reported skin lesions and $75 \%$ had vasoactive drugs, mainly Noradrenaline $(72.5 \%)$. Conclusion: Knowledge of the sociodemographic and health profile of these people is fundamental for the professionals working in an ICU, because, through the specific information, they can draw up strategies that allow an adequate and quality care for the population, in its specificity.

Key words: Intensive Care Units, Mortality, Sepsis.

1Universidade de Brasília (UNB), Brasília - DF. *E-mail: anaauroraluz@hotmail.com 


\section{RESUMEN}

Objetivo: Identificar el perfil sociodemográfico y de salud de los pacientes que evolucionaron a la muerte en la Unidad de Terapia Intensiva (UTI) de un hospital universitario. Método: Estudio transversal, retrospectivo, descriptivo, con abordaje cuantitativo, basado en datos secundarios extraídos en análisis de prontuarios. La muestra fue compuesta por todos los prontuarios de pacientes que evolucionaron a muerte en la UTI durante el año $2015(n=40)$. Resultados: Se constató que 20,7\% de los pacientes internados evolucionaron a óbito, en su mayoría ancianos (67,5\%), del sexo masculino (67,5\%); El 30\% procedía del centro quirúrgico, con una media de 22,6 días de internación; El 75\% de los diagnósticos de defunción fueron sepsis / shock séptico, presentaron en promedio 6,3 procedimientos invasivos por paciente, 80\% tenían relato de lesión en piel y $75 \%$ hicieron uso de drogas vasoactivas, principalmente la Noradrenalina (72,5\%). Conclusión: El conocimiento del perfil sociodemográfico y de salud de esas personas es fundamental para los profesionales que trabajan en una UTI, pues, a través de las informaciones específicas, pueden trazar estrategias que posibiliten un cuidado adecuado y de calidad para la población, en su especificidad.

Palabras clave: Unidades de Cuidados Intensivos, Mortalidad, Sepsis.

\section{INTRODUÇÃO}

A Unidade de Terapia Intensiva (UTI) é uma unidade hospitalar de extrema importância, pois é nela que acontece $\mathrm{o}$ atendimento de pacientes graves, realizado por profissionais especializados, de forma contínua, utilizando materiais específicos e tecnologias necessárias ao diagnóstico, monitorização e terapia (BRASIL, 2010). Essa unidade utiliza muitos recursos técnicos e novas tecnologias que têm a capacidade de manter a sobrevida do paciente, até que este possa se recuperar (MELO EM, et al., 2015).

Os profissionais que trabalham com terapia intensiva, precisam ter conhecimento suficiente e habilidade para prestar esse tipo de cuidado. Embora a UTI seja a unidade que atende pacientes graves que irão se recuperar, nem todos têm o mesmo destino. Muitos desses pacientes internados numa UTI já estão ou evoluem para um estágio terminal de vida (SANCHES PG e CARVALHO MDB, 2009).

A Resolução da Agência Nacional de Vigilância Sanitária (ANVISA), № 7, de 24 de fevereiro de 2010, define paciente crítico/grave como:

“... paciente com comprometimento de um ou mais dos principais sistemas fisiológicos, com perda de sua autorregulação, necessitando de assistência contínua" (BRASIL, 2010).

O paciente internado em uma UTI, na grande maioria das vezes, tem sua melhora vinculada à utilização de procedimentos invasivos. Geralmente, as UTIs são dotadas de suportes tecnológicos avançados que visam a manutenção da vida, como por exemplo, ventiladores mecânicos, dispositivos invasivos, fármacos potentes e dispositivos eletrônicos, que serão utilizados pela equipe capacitada para tal (NEEDHAM DM, et al., 2005; FAVARIN S e CAMPONOGARA S, 2012).

Assim, o parâmetro para um paciente permanecer na UTI é o seu quadro clínico, o qual irá demandar um acompanhamento médico contínuo por conta da sua instabilidade e risco de morte. A equipe multiprofissional deve ter capacidade para tomar decisões rapidamente, visando otimizar o tempo do cuidado prestado ao paciente (NEEDHAM DM, et al., 2005). Em se tratando de pacientes graves, o tempo é crucial.

Ademais, na UTI, os pacientes estão acamados por tempo prolongado, grande parte imunodeprimidos e com doenças graves que necessitam de antibióticos potentes e de monitorização invasiva, o que os leva a ser mais vulneráveis às infecções hospitalares (TEJEDOR SC, et al., 2012; SANTOS AV, et al., 2016).

Por esta razão, a UTI, geralmente, é um setor que tende a ter alto número de óbitos. Obter dados sociodemográficos e epidemiológicos dos pacientes internados na UTI possibilita traçar novas estratégias para prestar um melhor cuidado a esses pacientes (RODRIGUEZ AH, et al., 2016). 
Espera-se, com este estudo, contribuir com a literatura científica sobre o tema, proporcionar informações para a equipe de saúde, especialmente os enfermeiros, para que possam se preparar melhor enquanto profissionais e traçar estratégias de cuidado, para os pacientes e seus familiares.

Para isso, o presente estudo tem como objetivo, caracterizar o perfil sociodemográfico e de saúde dos pacientes que evoluíram a óbito na UTI de um hospital universitário.

\section{MÉTODOS}

Trata-se de um estudo transversal, retrospectivo, descritivo, com abordagem quantitativa, baseado na análise de prontuários e coleta de dados secundários. O estudo foi desenvolvido em um hospital universitário do Distrito Federal, no Serviço de Arquivo Médico (SAME), dessa instituição.

A amostra do estudo foi composta por todos os prontuários de pacientes que evoluíram a óbito na UTI durante $o$ ano de 2015. Para determinar a amostra, foi considerada a amostragem não-probabilística, do tipo amostra por conveniência.

Os critérios de inclusão foram: prontuários de pacientes de ambos os sexos, admitidos e que evoluíram a óbito na UTI durante o ano de 2015, no período de primeiro de janeiro a 31 de dezembro. Como critério de exclusão, todos os prontuários não disponíveis no dia de coleta de dados.

A coleta de dados foi realizada pelas autoras do presente estudo. Deu-se por meio da busca e análise de prontuários dos pacientes, tendo um questionário auxiliar de pesquisa, como guia de registro desses dados. O questionário foi construído pelos autores do presente estudo com base em informações contidas em publicações sobre o tema.

A seleção dos prontuários foi feita de acordo com o caderno de registro de admissão de pacientes da UTI. A UTI em questão tinha 10 leitos ativos, para pessoas com idade acima de 15 anos. Dos 196 pacientes internados na UTI em 2015, 40 evoluíram a óbito. Os 40 prontuários estavam disponíveis para consulta.

Os dados deste estudo são descritivos. Para descrever o perfil da amostra, foram elaboradas tabelas de frequência das variáveis categóricas, com valores de frequência absoluta (n), percentual (\%), assim como estatísticas descritivas das variáveis ordinais.

O presente estudo atendeu à Resolução CNS 466/2012. O projeto foi aprovado pelo Comitê de Ética da Faculdade de Ciências da Saúde da Universidade de Brasília, sob o CAAE: 64108417.1.0000.0030, parecer $\mathrm{n}^{0}$ 2.197.661. Foram utilizados apenas e exclusivamente dados secundários dos prontuários de pacientes. Cada prontuário recebeu um número e os dados foram analisados de forma agrupada.

\section{RESULTADOS}

Os dados sociodemográficos dos 40 pacientes que evoluíram a óbito na UTI (20,7\%) estão apresentados na Tabela 1.

A idade das pessoas que morreram na UTI, em 2015, variou entre 17 e 81 anos, com média de 61,1 anos. A maioria tinha idade $\geq 60$ anos $(67,5 \%)$, era do sexo masculino (67,5\%) e casados (37,5\%) (Tabela 1).

Não foram encontradas informações sobre a situação ocupacional dessas pessoas em $47,5 \%$ dos prontuários. Nos prontuários que continham tal informação, $27,5 \%$ dos pacientes trabalhavam antes da internação e $25 \%$ eram aposentados.

Quanto à escolaridade, 15\% tinham quatro anos de estudo e também 15\% dos pacientes tinham 11 anos ou mais de estudo. Em $57,5 \%$ dos prontuários não havia informação quanto à escolaridade. Grande parte desses pacientes era proveniente do Nordeste (32,5\%).

Em relação à residência, $67,5 \%$ deles residiam no DF. Ao buscar informações sobre a renda pessoal e familiar, nenhum dado foi encontrado nos prontuários (Tabela 1). 
Tabela 1 - Distribuição das variáveis sociodemográficas dos prontuários dos indivíduos internados na UTI, em 2015, que foram a óbito $(n=40)$, Brasília, DF, 2018.

\begin{tabular}{|c|c|c|}
\hline Variáveis & $\mathbf{n}$ & $\%$ \\
\hline \multicolumn{3}{|l|}{ Idade (anos) } \\
\hline Até 19 & 1 & 2,5 \\
\hline De 20 a 39 & 3 & 7,5 \\
\hline De 40 a 59 & 9 & 22,5 \\
\hline Maior ou igual a 60 & 27 & 67,5 \\
\hline \multicolumn{3}{|l|}{ Sexo } \\
\hline Masculino & 27 & 67,5 \\
\hline Feminino & 13 & 32,5 \\
\hline \multicolumn{3}{|l|}{ Situação ocupacional } \\
\hline Trabalhadores & 11 & 27,5 \\
\hline Aposentado & 10 & 25,0 \\
\hline Pensionista & 0 & 0,0 \\
\hline Não informado & 19 & 47,5 \\
\hline \multicolumn{3}{|l|}{ Estado Civil } \\
\hline Casado/ União Estável & 16 & 40,0 \\
\hline Solteiro & 14 & 35,0 \\
\hline Separado/Divorciado & 3 & 7,5 \\
\hline Viúvo & 2 & 5,0 \\
\hline Não informado & 5 & 12,5 \\
\hline \multicolumn{3}{|l|}{ Escolaridade (anos) } \\
\hline 0 & 1 & 2,5 \\
\hline 4 & 6 & 15,0 \\
\hline 8 & 4 & 10,0 \\
\hline$\geq 11$ & 6 & 15,0 \\
\hline Não informado & 23 & 57,5 \\
\hline \multicolumn{3}{|l|}{ Local de origem } \\
\hline Nordeste & 13 & 32,5 \\
\hline Sudeste & 9 & 22,5 \\
\hline Goiás & 6 & 15,0 \\
\hline Distrito Federal & 5 & 12,5 \\
\hline Outros & 4 & 10,0 \\
\hline \multicolumn{3}{|l|}{ Local de Residência } \\
\hline Distrito Federal & 27 & 67,5 \\
\hline Goiás & 12 & 30,0 \\
\hline Morador de Rua & 1 & 2,5 \\
\hline
\end{tabular}

Fonte: Ferrão AARCN, et al., 2017.

Os dados relacionados à saúde estão apresentados na Tabela 2. Dos pacientes em estudo, ao serem internados na UTI, 30\% vieram do centro cirúrgico e $25 \%$ eram oriundos da Unidade de Paciente Crítico (UPC)/Centro de Pronto Atendimento (CPA), ambas unidades do hospital em que foi realizada a pesquisa (Tabela 2).

Em relação ao mês de internação dessas pessoas, houve destaque para o primeiro e segundo trimestres de 2015: 37,5\% e 35\%, respectivamente. Além disso, os pacientes ficaram internados entre um dia (tempo mínimo) e 121 dias (tempo máximo), com média de 22,6 dias de internação. Houve predomínio de 11 a 30 dias de internação (37,5\%). Sete desses pacientes (17,5\%) ficaram internados por mais de um mês (Tabela 2).

Segundo os diagnósticos dos óbitos, registrados nos atestados de óbito de cada paciente, 75\% deles indicaram como causa sepse/choque séptico. Além disso, dentre as outras causas de óbito, destacaram-se outros tipos de choque $(10 \%)$, insuficiência respiratória $(7,5 \%)$ e outros $7,5 \%$ morreram por outras causas (Tabela 2). Em 95\% dos prontuários não havia informação a respeito do tempo de espera dos pacientes para efetivação de vaga na UTI. 
Tabela 2 - Distribuição das variáveis em relação à internação dos indivíduos na UTI, em 2015, que foram a óbito ( $\mathrm{n}=40)$, Brasília, DF, 2018.

\begin{tabular}{|c|c|c|}
\hline Variáveis & $\mathbf{n}$ & $\%$ \\
\hline \multicolumn{3}{|l|}{ Procedência antes da UTI } \\
\hline Centro Cirúrgico & 12 & 30,0 \\
\hline UPC/CPA & 10 & 25,0 \\
\hline Clínica Cirúrgica & 4 & 10,0 \\
\hline Clínica Médica & 2 & 5,0 \\
\hline Transplante & 2 & 5,0 \\
\hline Outros & 8 & 20,0 \\
\hline Não informado & 2 & 5,0 \\
\hline \multicolumn{3}{|c|}{ Trimestre de Internação na UTI } \\
\hline Trimestre 1 & 15 & 37,5 \\
\hline Trimestre 2 & 14 & 35,0 \\
\hline Trimestre 3 & 6 & 15,0 \\
\hline Trimestre 4 & 5 & 12,5 \\
\hline \multicolumn{3}{|l|}{ Período de Internação } \\
\hline Até 5 dias & 13 & 32,5 \\
\hline 6 a 10 dias & 5 & 12,5 \\
\hline 11 a 30 dias & 15 & 37,5 \\
\hline Mais de 31 dias & 7 & 17,5 \\
\hline \multicolumn{3}{|l|}{ Causa da morte } \\
\hline Sepse/choque séptico & 30 & 75,0 \\
\hline Outros tipos de Choque & 4 & 10,0 \\
\hline Insuficiência Respiratória & 3 & 7,5 \\
\hline Outros & 3 & 7,5 \\
\hline
\end{tabular}

Fonte: Ferrão AARCN, et al., 2017.

Os demais dados relacionados à saúde, referentes aos prontuários dos pacientes internados na UTI em 2015, estão apresentados na Tabela 3. Quanto aos antecedentes diagnósticos, $62,5 \%$ dos casos tinham relato de Hipertensão Arterial Sistêmica (HAS), 30,0\% de doença renal, 27,5\% de Diabetes Mellitus (DM) e 20,0\% de sepse/choque séptico (Tabela 3 ).

Dentre os motivos de internação, os principais foram pós-operatórios (55\%) e sepse/choque séptico (37,5\%). Durante a internação, 60\% apresentaram diagnóstico de sepse/choque séptico e o principal foco de infecção foi o pulmonar (27,5\%) (Tabela 3). Quanto aos óbitos na UTI, 92,5\% deles eram relativos a pacientes internados pela primeira vez.

Em relação à visita de familiares, apenas $20 \%$ dos prontuários tinham essa informação registrada. $\mathrm{O}$ grau de parentesco mais comum, quando registrado, foi cônjuge (25\%) e filhos (25\%). A maior parte dos pacientes não teve hospitalização nos últimos 12 meses que antecederam a internação na UTI.

Quanto aos procedimentos realizados nesses pacientes, 97,5\% fizeram uso de cateter venoso central, $92,5 \%$ de cateter vesical de demora, $87,5 \%$ de ventilação mecânica, 85\% de pressão arterial invasiva, 72,5\% de nutrição enteral, $65 \%$ de hemodiálise, $67,5 \%$ de traqueostomia, 30\% de dreno de tórax, 30\% de outros tipos de dreno, e 12,5\% de nutrição parenteral (Tabela 3).

Quando avaliados individualmente pelo número de procedimentos invasivos aos quais foram submetidos durante a internação na UTI, pode-se verificar que, em média, cada pessoa fez uso de 6,3 procedimentos, variando de três a 10 deles. Houve predomínio dos pacientes com três a seis procedimentos invasivos: 21 $(52,5 \%)$ (Tabela 3).

Ressalta-se que 32 pacientes (80\%) apresentaram história de lesão cutânea, sendo que 27,5\% eram lesão por pressão, com maior prevalência em região sacral (20\%). Dos 40 pacientes que evoluíram a óbito, $75 \%$ deles fizeram uso de drogas vasoativas, predominantemente a noradrenalina (72,5\%) (Tabela 3). Em relação à existência de relato de câncer, em 15 (37,5\%) deles havia descrição desse diagnóstico. 


\section{Revista Eletrônica Acervo Saúde / Electronic Journal Collection Health ｜ ISSN 2178-2091}

Tabela 3 - Distribuição das variáveis clínicas dos prontuários dos indivíduos internados na UTI, em 2015, que foram a óbito (n=40), Brasília, DF, 2018.

\section{Variáveis \\ Antecedentes Diagnósticos*}

$\mathbf{n}$

$$
\text { HAS }
$$

Doença Renal

DM

Sepse/Choque séptico

PNM

Outros

Não informado

25

$25 \quad 62,5$

$\begin{array}{lr}12 & 30\end{array}$

$11-27,5$

$\begin{array}{cc}11 & 20\end{array}$

$5 \quad 12,5$

$\begin{array}{lr}5 & 60\end{array}$

\section{Motivo da Internação*}

Pós-operatório

1

Sepse/Choque séptico

Insuficiência Renal

2,5

Insuficiência Respiratória

Outros

$22-55$

$\begin{array}{cc}22 & 55 \\ 15 & 37,5\end{array}$

$\begin{array}{ll}9 & 37,5 \\ 5 & 22,5\end{array}$

Outros

Sepse/choque séptico durante a internação

Não

Não informado

32

12,5

80

Foco da Infecção

Pulmonar 11

Abdominal

Urinário

Outros

Não informado

$24 \quad 60$

$10-25$

$6 \quad 15$

Procedimentos realizados na UTI*

Cateter Venoso Central

11227,5

Cateter Vesical de Demora

Ventilação Mecânica

$\begin{array}{lc}4 & 10 \\ 2 & 5 \\ 3 & 7,5\end{array}$

Pressão Arterial Invasiva

Nutrição Enteral

4

1

39

97,5

92,5

87,5

$35 \quad 87,5$

$34 \quad 85$

72,5

REAS/EJCH | Vol.12(8) | e3509 | DOI: https://doi.org/10.25248/reas.e3509.2020 Página 6 de 11 


\section{Revista Eletrônica Acervo Saúde / Electronic Journal Collection Health ｜ ISSN 2178-2091}

\begin{tabular}{|c|c|c|}
\hline Variáveis & $\mathbf{n}$ & $\%$ \\
\hline \multicolumn{3}{|c|}{ Procedimentos realizados na UTI* } \\
\hline Traqueostomia & 23 & 67,5 \\
\hline Hemodiálise & 26 & 65 \\
\hline Dreno de Tórax & 12 & 30 \\
\hline Outros tipos de dreno & 12 & 30 \\
\hline Nutrição Parenteral & 5 & 12,5 \\
\hline \multicolumn{3}{|c|}{ Procedimentos invasivos (unidade) } \\
\hline $3-6$ & 21 & 52,5 \\
\hline 7-10 & 19 & 47,5 \\
\hline \multicolumn{3}{|l|}{ Lesão em pele } \\
\hline Sim & 32 & 80 \\
\hline Não & 6 & 15 \\
\hline Não informado & 2 & 5 \\
\hline \multicolumn{3}{|l|}{ Lesão por pressão } \\
\hline Sim & 11 & 27,5 \\
\hline Não & 26 & 65 \\
\hline Não informado & 3 & 7,5 \\
\hline \multicolumn{3}{|c|}{ Local da lesão por pressão* } \\
\hline Sacral & 8 & 20 \\
\hline Glúteos & 2 & 5 \\
\hline Trocantérica & 1 & 2,5 \\
\hline Não informado & 1 & 2,5 \\
\hline \multicolumn{3}{|c|}{ Uso de Drogas Vasoativas (DVA) } \\
\hline Sim & 30 & 75 \\
\hline Não & 9 & 22,5 \\
\hline Não informado & 1 & 2,5 \\
\hline
\end{tabular}

${ }^{*}$ Os pacientes podiam apresentar mais de uma resposta

Fonte: Ferrão AARCN, et al., 2017. 


\section{DISCUSSÃO}

Identificou-se que $20,7 \%$ dos pacientes internados na UTI evoluíram a óbito. Dados semelhantes (20,4\%) foram encontrados em estudo realizado com uma população maior (RODRIGUEZ AH, et al., 2016). Já em um outro estudo com 104 prontuários analisados, $50 \%$ dos pacientes foram a óbito (FAVARIN S e CAMPONOGARA S, 2012).

A maioria dos pacientes internados em UTI era de idosos, ou seja, tinha idade maior ou igual a 60 anos, dado que corrobora um estudo que também mostra que a faixa etária predominante da população internada na UTI era a de idoso. Segundo o referido estudo, dos 104 prontuários analisados, 26\% dos pacientes eram da faixa etária entre 61 a 70 anos e 15\% eram da faixa etária de 71 a 80 anos (FAVARIN S e CAMPONOGARA S, 2012).

Sabe-se que, quanto maior a idade, mais frágil tende a ser a saúde dessas pessoas. Assim, há necessidade de maior suporte médico e de cuidado em geral. Além disso, a recuperação de pessoas idosas é mais difícil do que a de pessoas mais jovens (OLIVEIRA VCR, et al., 2011) o que poderia justificar a maior mortalidade de pessoas idosas.

O sexo masculino prevaleceu nos dados do presente estudo, assim como em outros estudos sobre o tema (SCHEIN LEC e CESAR JA, 2010; FAVARIN S e CAMPONOGARA S, 2012; FRANÇA CDM, et al., 2013; CAMUCI MB, et al., 2014; RODRIGUEZ AH, et al., 2016; ORBAN JC, et al., 2017). Essa predominância do sexo masculino pode estar associada ao comportamento dos homens, que, em geral, só procuram assistência médica quando já estão com quadros mais graves de saúde (RODRIGUEZ AH, et al., 2016).

Vale destacar que em $47,5 \%$ dos prontuários não constavam, nem a informação acerca da situação ocupacional dos pacientes internados na UTI, nem informações sobre renda pessoal e familiar. Esses dados são importantes, pois possibilitam conhecer aspectos econômicos, financeiros e sociais da pessoa e, até mesmo, identificar o quanto esses aspectos influenciam no âmbito familiar.

Nesse sentido, o conhecimento desses dados permite viabilizar os encaminhamentos aos serviços assistenciais necessários, facilitando o contato entre a família e a instituição hospitalar. Permite também agilizar os trâmites necessários, relacionados ao trabalho/ocupação de quem foi internado, amenizando possíveis riscos de prejuízo para o paciente e sua família.

No que diz respeito ao estado civil dos pacientes do presente estudo, foi evidenciado que 37,5\% deles eram casados. Estudo realizado especificamente com idosos demonstrou que $50,2 \%$ eram casados (SCHEIN LEC e CESAR JA, 2010).

Em relação à escolaridade, vale destacar que $57,5 \%$ dos prontuários não continha essa informação. Porém, trata-se de um dado essencial para que a equipe de saúde conheça o seu público de atendimento (tanto pacientes quanto visitantes), saiba como se dirigir aos pacientes e que tipo de linguagem usar para que as informações que precisam ser passadas sejam devidamente compreendidas pelas partes interessadas. $O$ nível de escolaridade pode ser um obstáculo na comunicação entre o paciente e sua equipe de saúde, pois o paciente pode não se sentir à vontade para expor suas dúvidas e angústias (VICTOR ACS, et al., 2003).

Quanto à proveniência, a maioria dos pacientes que foram a óbito $(32,5 \%)$ era originária do Nordeste. Sabe-se que existe grande evasão de pessoas do Nordeste para outros estados, o que mostra que as pessoas nessa situação procuravam uma melhor condição de vida, oportunidades de trabalho, melhores atendimentos na área da saúde, entre outros. Além disso, Brasília se destaca, pois é uma cidade em que muitas pessoas vieram de diferentes lugares, e principalmente do Nordeste, para a sua construção, aqui viveram e construíram suas famílias (CARVALHO ARC, 2008). A grande maioria dos pacientes $(67,5 \%)$ do presente estudo residia no DF e em Goiás. Dados semelhantes foram encontrados em estudo também realizado no DF (GUIA MC, et al., 2015).

Em relação à origem dos pacientes que foram internados na UTI e que evoluírama óbito, a maioria foi proveniente do centro cirúrgico (30\%). Dados similares foram encontrados em um estudo no qual $52,5 \%$ dos pacientes também saíram do Centro Cirúrgico para a UTI (RODRIGUEZ AH, et al., 2016). 
Em se tratando do tempo de internação na UTI, achados na literatura mostram que as internações podem durar de 0 a 73 dias, com tempo médio de 6 dias (RODRIGUEZ AH, et al., 2016). Outro estudo evidenciou que o tempo médio de internação foi de 14 dias, sendo que o maior tempo de internação foi de 95 dias (FAVARIN S e CAMPONOGARA S, 2012). Os dados apresentados, independentemente do desfecho das internações, aproximaram-se dos dados encontrados neste estudo, em que o tempo mínimo de internação foi de um dia e o tempo máximo foi de 121 dias, com média de 22,6 dias duração.

A principal causa de óbito foi sepse/choque séptico (75\%), o que corrobora estudos que obtiveram resultados de que a maioria dos óbitos foi vinculada às doenças do aparelho circulatório (RODRIGUEZ AH, et al., 2016). Um dos estudos refere que choque séptico e sepse foram evidenciados dentre as morbidades que mais foram adquiridas durante a internação da população estudada, mas não indica diretamente se essas morbidades foram causas de óbitos (FAVARIN S e CAMPONOGARA S, 2012).

O choque séptico é o nível mais grave que a sepse pode atingir. É um estado de hipoperfusão tecidual que é causado por algum microrganismo infectante, situação essa que leva à necessidade de fazer uso de drogas vasopressoras. Infelizmente, o choque séptico ainda é a principal causa de óbito em UTIs que tratam de adultos. O prognóstico ainda é desfavorável, mesmo com tantas inovações (SANTOS AV, et al., 2015).

Em UTIs é comum existirem casos de infecções hospitalares, pois é um ambiente com pacientes graves que necessitam de muitos procedimentos invasivos e de uso de antibióticos. Estes procedimentos podem ser porta de entrada de agentes infecciosos e os antibióticos podem levar ao aparecimento de microrganismos multirresistentes, respectivamente (SANTOS AV, et al., 2015).

Nesse sentido, o achado neste estudo é bastante expressivo e de grande importância. De certa forma, é um resultado preocupante, pois pode sugerir a ocorrência de casos de infecção hospitalar, que é uma situação que exige atenção redobrada dos profissionais da UTI. É essencial que sejam implantados protocolos para detectar e tratar a sepse, que os profissionais sigam à risca todos os protocolos de controle de infecções, e tenham um grande cuidado ao realizarem técnicas que exigem esterilidade (SANTOS AV, et al., 2015).

Em $72,5 \%$ dos prontuários não havia registro a respeito de visitas ao paciente internado na UTI. Porém, esse é um dado relevante a ser registrado, pois a presença da família durante o tratamento/internação do paciente é muito importante. Pode auxiliar no êxito do tratamento e facilitar a comunicação entre o paciente e a equipe de saúde.

Caso não haja a presença da família, é possível que o ser humano, como ser biopsicossocial, tenha a sua parte psicológica abalada, interferindo no seu processo de cura. Nesse sentido, estudos mostram que o toque humano é capaz de desacelerar os batimentos cardíacos do paciente (BECCARIA LM, et al., 2008).

Os principais antecedentes diagnósticos dos pacientes internados foram HAS (62,5\%), Doença Renal (30\%), DM (27,5\%) e SEPSE/Choque Séptico (20\%). A principal causa de morte, no Brasil, são as doenças não transmissíveis $(74 \%)$, sendo que, dentre elas, as principais são as doenças cardiovasculares (destacando-se isquemias e doenças cerebrovasculares), seguidas de câncer, diabetes, doenças do aparelho respiratório e doenças do aparelho digestivo (REIS C, et al., 2016; SILVA JBVB, et al., 2018).

Pode-se ver acima que o antecedente diagnóstico mais comum neste estudo foi a HAS, fazendo jus ao que foi achado no referido artigo; e o diabetes mellitus também vem em terceiro lugar, mostrando que são morbidades muito comuns nos brasileiros.

Os principais motivos de internação foram pós-operatórios (55\%) e quadros de sepse/choque séptico $(37,5 \%)$. São vários os motivos que levam a uma internação em uma UTI. Dentre eles está o pós-operatório, internação essa que visa a recuperação desse paciente e, também, 0 atendimento rápido a possíveis complicações (BALSANELLI AP, et al., 2006). Rodriguez AH et al. (2016) afirma em seu estudo que a principal causa de internação na UTI foi de doenças do aparelho circulatório $(23,3 \%)$.

Favarin S e Camponogara S (2012) afirmam que o principal motivo de internação foram doenças infecciosas (28\%). A sepse/choque séptico se encaixa em ambas as classificações, pois é um quadro infeccioso grave, quadro esse que interfere diretamente no aparelho circulatório. Apesar de, nesse estudo, a 
sepse/choque séptico aparecer em segundo lugar, as porcentagens são parecidas quando comparadas aos estudos desses autores.

O principal foco de sepse/choque séptico na UTI foi o pulmonar, fato também constatado em outros trabalhos (SANTOS AV, et al, 2016; FAVARIN S e CAMPONOGARA S, 2012). Dentre os procedimentos realizados, houve destaque para a realização de Cateterização Venosa Central, cateterização vesical de demora e ventilação mecânica.

Esses procedimentos acarretam riscos, tanto a nível local quanto a nível sistêmico, e exigem uma especial atenção no que diz respeito à justificativa de necessidade de sua inserção, manipulação e remoção (SANTOS AV, et al., 2016).

A maioria dos pacientes fez uso, em média, de 6,3 procedimentos invasivos. Pode- se entender, assim, que a quantidade desses procedimentos realizados em UTIs é muito grande, e esse fato faz com que os pacientes estejam mais susceptíveis a contraírem infecções, pois possuem mais portas de entrada de microorganismos (OLIVEIRA AC, et al., 2010). A grande quantidade de procedimentos invasivos realizados nas UTIs é uma das principais razões para o desenvolvimento de sepse/choque séptico em pacientes que necessitam de suporte intensivo (TELES LS, et al., 2017).

No que diz respeito às lesões de pele, $80 \%$ dos pacientes que morreram possuíam algum tipo de lesão. A pele é uma barreira natural do corpo humano, e uma lesão é resultado de sua ruptura, podendo assim se tornar porta de entrada para microrganismos. É fundamental que as feridas sejam avaliadas, procurando qualquer sinal de infecção, pois onde há tecidos não vitalizados, o ambiente se torna propício à presença de bactérias (PERUZZO AB, et al., 2005).

Dentre as lesões de pele, $27,5 \%$ dos pacientes que foram a óbito possuíam lesão por pressão. É importante ressaltar que essas lesões geram grandes custos para o hospital, pois possuem tratamento lento e difícil e, mais importante, aumentam a mortalidade desses pacientes (SILVA MLN, et al., 2013).

Em UTIs é comum que sejam prescritas drogas vasoativas (DVA) como parte do tratamento, pois estão internados pacientes graves e que muitas vezes necessitam de um reforço hemodinâmico. Em um estudo semelhante, foi obtido como resultado que $75,7 \%$ dos pacientes que foram a óbito daquele hospital estudado fizeram uso de DVAs, número esse muito parecido com o resultado deste estudo, no qual foi obtido que $75 \%$ dos pacientes fizeram uso de DVAs (MORITZ RD, et al. 2008). A droga vasoativa mais utilizada neste estudo foi a Noradrenalina (72,5\%), droga esta comumente utilizada no combate ao choque séptico (WESTPHAL GA, et al., 2011).

Em relação à presença de diagnóstico de câncer nos pacientes do presente estudo, apenas $37,5 \%$ apresentaram tal relato. Martelletti LBS et al. (2019) apresenta dado semelhante, no qual os pacientes com diagnóstico de câncer somam 40,9\%.

O câncer, além de ser uma doença com alto índice de mortalidade, também tem relação com a presença de sepse/choque séptico, principal causa de morte evidenciada neste estudo. Os fatores que fazem existir essa relação são vários, entre eles o tipo de terapia escolhida para tratamento, depressão do sistema imune, neutropenia, rupturas de barreiras cutâneas e mucosas, entre outros (RAVETTI CG, 2014).

\section{CONCLUSÃO}

Conhecer as características sociodemográficas e de saúde da população atendida na UTI é fundamental, pois assim é possível criar estratégias de cuidado para prestar um atendimento qualificado ao tipo de população predominante. A partir do conhecimento desses dados, os membros da equipe podem se preparar melhor enquanto profissionais, realizar eventos de educação permanente focalizados, promovendo a atualização do profissional e provendo um melhor atendimento ao paciente. Este estudo também é valoroso para contribuir com a literatura científica, que é escassa a respeito deste tema. Ressalta-se a importância de haver maior organização das informações em prontuários e registro cuidadoso de informações mais completas por toda a equipe de saúde. 


\section{REFERÊNCIAS}

1. BALSANELLI AP, et al. Carga de trabalho de enfermagem e sua relação com a gravidade dos pacientes cirúrgicos em UTI. Acta Paul Enferm., 2006; 19: 16-20.

2. BECCARIA LM, et al. Visita em Unidades de Terapia Intensiva: concepção dos familiares quanto à humanização do atendimento. Arq. Ciênc. Saúde, 2008, 15(2): 65-69, abr./jun.

3. BRASIL. Agência Nacional de Vigilância Sanitária (ANVISA). Resolução No 7, de 24 de fevereiro de 2010. Dispõe sobre os requisitos mínimos para funcionamento de Unidades de Terapia Intensiva e dá outras providências. Fev., 2010.

4. CAMUCI MB, et al. Caracterização epidemiológica de pacientes adultos internados em uma Unidade de Terapia Intensiva de queimados. Cogitare Enferm., 2014, 19: 78-83, jan./mar.

5. CARVALHO ARC. Migrantes em Brasília: os motivos, as dores e os sonhos numa perspectiva clínica. Dissertação de Mestrado. Universidade de Brasília, Brasília, 2008; $97 f$.

6. FAVARIN S, CAMPONOGARA S. Perfil dos pacientes internados na unidade de terapia intensiva adulto de um hospital universitário. Rev. Enferm. UFSM, 2012, 2(2): 320-329.

7. FRANÇA CDM, et al. Perfil epidemiológico da Unidade de Terapia Intensiva de um Hospital Universitário. InterScientia, João Pessoa, 2013, 1(2): 72-82, maio/ago.

8. GUIA MC, et al. Perfil epidemiológico e preditores de mortalidade de uma unidade de terapia intensiva geral de hospital público do Distrito Federal. Com. Ciências Saúde, 2015, 26(1/2): 9-19.

9. MARTELLETTI LBSJ, et al. Perfil sociodemográfico e clínico de pacientes com câncer internados em uma Unidade de Terapia Intensiva adulto. Revista Eletrônica Acervo Saúde, 2019, 11(13): e985, 31 ago.

10. MELO EM, et al. Clinical and demographic characteristics of patients on mechanical ventilation in the intensive care unit. Rev Enferm UFPI, 2015, 4(3): 36-41, Jul-Set.

11. MORITZ RD, et al. Avaliação dos óbitos ocorridos no hospital universitário da Universidade Federal de Santa Catarina (HU/UFSC). Rev. Assoc. Med. Bras., 2008, 54(5): 390-395.

12. NEEDHAM DM, et al. A system factors analysis of "line, tube, and drain" incidents in the intensive care unit. Crit. Care Med., 2005, 33: 1701-1707.

13. OLIVEIRA AC, et al. Infecção hospitalar em unidade de tratamento intensivo de um hospital universitário brasileiro. Rev. Latino-Am. Enfermagem, 2010, 18(2): [08 telas], mar.-abr.

14. OLIVEIRA VCR, et al. Evolução clínica de adultos, idosos e muito idosos internados em Unidade de Terapia Intensiva. Rev. Latino- Am. Enfermagem, 2011, 19(6): [8 telas], nov.-dez.

15. ORBAN JC, et al. Causes and Characteristics of Death in Intensive Care Units: A Prospective Multicenter Study. Anesthesiology 5, 2017, 126: 882-889.

16. PERUZZO AB, et al. Protocolo de cuidados a pacientes com lesão de pele. Mom. \& Perspec. Saúde, 2005, 18(2), Porto Alegre, jul./dez.

17. RAVETTI CG. Fatores preditores de mortalidade em pacientes oncológicos admitidos em unidade de terapia intensiva com sepse grave e choque séptico. Tese de Doutorado. Universidade Federal de Minas Gerais, Belo Horizonte, 2014; 93f.

18. REIS C, et al. O desafio do envelhecimento populacional na perspectiva sistêmica de saúde. BNDES Setorial, 2016, 44: [87]-124, Rio de Janeito, set.

19. RODRIGUEZ AH. Epidemiological characteristics and causes of deaths in hospitalized patients under intensive care. Rev. Bras. Enferm. [Internet], 2016 , 69(2): 210-214.

20. SANCHES PG, CARVALHO MDB. Vivência dos enfermeiros de unidade de terapia intensiva frente à morte e o morrer. Rev. Gaúcha Enferm., 2009, 30(2): 289-296, Porto Alegre: RS, jun.

21. SANTOS AV, et al. Perfil epidemiológico da sepse em um hospital de urgência. Rev. Prevenção de Infecção e Saúde (REPIS), 2015, 1(1).

22. SANTOS AV, et al. Perfil das infecções hospitalares nas unidades de terapia intensiva de um hospital de urgência. Rev. Enferm. UFPE on-line, 2016, 10(Supl. 1): 194-201, Recife, jan.

23. SCHEIN LEC, CESAR JA. Perfil de idosos admitidos em unidades de terapia intensiva gerais em Rio Grande, RS: resultados de um estudo de demanda. Rev. Bras. Epidemiol., 2010, 13(2): 289-301.

24. SILVA JBVB, et al. Perfil clínico de longevos em uma unidade de terapia intensiva. Acta Paul Enferm, 2018, 31(1): 39-45.

25. SILVA MLN, et al. Úlcera por pressão em Unidade de Terapia Intensiva: análise da incidência e lesões instaladas. Rev. Rene., 2013, 14(5): 938-944.

26. TEJEDOR SC, et al. Temporary central venous catheter utilization patterns in a large tertiary care center: tracking the "idle central venous catheter". Infect Control Hosp. Epidemiol. [Internet], 2012, 33: 50-57.

27. TELES LS, et al. Enfermagem frente à sepse: uma revisão literária. INTERNATIONAL NURSING CONGRESS Theme: Good practices of nursing representation in the construction of society, 2017: 9-12, maio.

28. VICTOR ACS, et al. Comunicação verbal de uma equipe médica: percepções e necessidades de visitantes de uma UTI. Acta Scientiarum Health Sciences, 2003, 25(2): 199-206, Maringá.

29. WESTPHAL GA, et al. Diretrizes para o tratamento da sepse grave/choque séptico - ressuscitação hemodinâmica. Revista Brasileira de Terapia Intensiva, 2011, 23(1): 13-23, Ribeirão Preto. 